\title{
Primeiro relato de Aetalion reticulatum (L.) (Hemiptera: Aetalionidae) infestando plantas de noni [Morinda citrifolia L. (Rubiaceae)]
}

\author{
Marcelo Tavares de Castro ${ }^{\circledR}$ \& Sandro Coelho Linhares Montalvão²
}

1. Faculdade ICESP/Promove de Brasília. 2. Embrapa Recursos Genéticos e Biotecnologia, Brasília, Distrito Federal.

\section{EntomoBrasilis 12 (2): 81-83 (2019)}

\begin{abstract}
Resumo. Aetalion reticulatum (L.) (Hemiptera: Aetalionidae), uma cigarrinha altamente polífaga e reconhecidamente praga de várias culturas agrícolas e florestais, é relatada pela primeira vez infestando plantas de noni, Morinda citrifolia L. (Gentianales: Rubiaceae), em Brasília, Distrito Federal, Brasil. Esse novo registro aumenta a lista de hospedeiras do inseto, reforçando a necessidade de estudos mais aprofundados sobre essa cigarrinha e o seu controle.
\end{abstract}

Palavras-Chave: Cigarrinha; Inseto Fitófago; Plantas Medicinais; Praga Agrícola.

\section{First report of Aetalion reticulatum (L.) (Hemiptera: Aetalionidae) infesting plants of noni [Morinda citrifolia L. (Rubiaceae)]}

\begin{abstract}
Aetalion reticulatum (L.) (Hemiptera: Aetalionidae), a highly polyphagous leafhooper and known to be pest of various agricultural and forestry crops, is reported for the first-time infesting noni plants, Morinda citrifolia L. (Gentianales: Rubiaceae), in Brasília, Federal District, Brazil. This new record increases the host's list of this insect, reinforcing the necessity for more in-depth studies of this leafhooper and its control.
\end{abstract}

Keywords: Agricultural Pest; Leafhooper; Medicinal Plants; Phytophagous Insect.

MYlons orinda citrifolia L. (Gentianales: Rubiaceae), popularmente conhecida como noni, é uma planta arbustiva de origem asiática que atualmente está distribuída por vários continentes, incluindo o americano (CORREIA et al. 2011). No Brasil, a espécie é pouco conhecida e o seu cultivo ainda não é feito em escala comercial (ToMBolato et al. 2005). Seus frutos são ovalados, suculentos, com muitas sementes (Silva et al. 2012) e são utilizados de forma medicinal em diversas partes do mundo, com efeitos relacionados a atividades antifúngicas, antibióticas, anti-inflamatórias, entre outros (SolOMON 1999; WANG et al. 2002; Silva et al. 2012; Matoso et al. 2013; Pimentel et al. 2016). Porém, seu uso medicinal ainda está cercado de mitos e verdades. Portanto, produtos contendo noni no Brasil não devem ser comercializados, sendo necessários estudos mais aprofundados sobre as suas reais propriedades (MATOso et al. 2013; Pimentel et al. 2016).

Praticamente não há relatos de insetos causando danos em árvores de noni no Brasil (SousA et al. 2010), com exceção do registro de ataque por formigas da subespécie Atta sexdens rubropilosa Forel (Hymenoptera: Formicidae) no município de Colorado do Oeste, Rondônia (MATTE et al. 2016). Portanto, esse trabalho teve como objetivo registrar a ocorrência de Aetalion reticulatum (L.) (Hemiptera: Aetalionidae) infestando plantas de noni na região de Brasília, Distrito Federal, Brasil.

Cigarrinhas infestando plantas de noni foram observadas e coletadas de março a maio de 2018 em Brasília, Distrito Federal, Brasil ( $15^{\circ} 43^{\prime} 49.8^{\prime \prime} \mathrm{S} 47^{\circ} 53^{\prime} 59.1^{\prime} \mathrm{W}$ ). Alguns insetos foram transferidos para tubos Falcon contendo álcool $70 \%$ para posterior confirmação da espécie, com base na literatura e comparação com descrições em artigos (BORROR \& DELONG 1969; Costa Lima 1942; SANTOS et al. 2015; ZANUNCiO et al. 2015). Durante os meses de março a maio, foram realizadas visitas às plantas de noni com o propósito de verificar a ocorrência e o desenvolvimento dos insetos nas plantas, como a oviposição, ninfas e adultos. Além dos Aetalionidae, algumas formigas e abelhas foram observadas associadas às colônias das cigarrinhas.

As cigarrinhas foram identificadas como A. reticulatum. As plantas analisadas estavam com frutos (Figura 1A e 1B) e foram encontrados ovos, ninfas e adultos do inseto, o que indica que este pode completar todo o seu ciclo nessa planta hospedeira, que tem duração de aproximadamente 110 dias (GALlo et al.
Edited by:

Anderson Gonçalves da Silva

Article History:

Received: $30 . v .2018$

Accepted: $14 . i v .2019$
Corresponding author:

Marcelo Tavares de Castro

乃 marceloengflorestal@gmail.com

(1) https://orcid.org/0000-0002-9147-8039
Funding agencies:

$\checkmark$ Without funding declared 
2002). Os insetos foram encontrados principalmente nos ramos mais novos da planta e próximos aos frutos (Figura 1C). As ninfas possuíam corpo com coloração acinzentada com manchas amareladas no abdome (Figura 1D). Os adultos mediam de 9 a $11 \mathrm{~mm}$ de comprimento, com coloração amarronzada e asas com veias branco-esverdeadas e salientes (Figura $1-\mathrm{E}$ ). As fêmeas encontradas possuíam o hábito de proteger os ovos, e, mesmo quando sofriam injúrias, não os abandonavam, fato também observado por RANDO \& LIMA (2010).

Das cinco plantas avaliadas, todas apresentaram infestações pela cigarrinha próximas aos frutos, em número expressivo de indivíduos, incluindo todas as fases de desenvolvimento do inseto. Segundo Gallo et al. (2002), altas infestações de A. reticulatum podem resultar na morte do hospedeiro, pois tanto as ninfas quanto os adultos sugam a seiva da planta, prejudicando o seu desenvolvimento, principalmente de frutos e brotações novas.

Essa cigarrinha já foi relatada em diversas plantas nativas e cultivadas, como o algodoeiro [Gossypium hirsutum L. (Malvales: Malvaceae)], cafeeiro [Coffea sp. (Gentianales: Rubiaceae)], eucalipto [Eucalyptus sp. (Myrtales: Myrtaceae), acácia negra (Acacia mearnsii De Wild. (Fabales: Fabaceae)], Citrus sp. (Sapindales: Rutaceae) (SANTANA et al. 2005), manga [Mangifera indica L. (Sapindales: Anacardiaceae)] (NASCIMENTO \& CARVAlHo 1998), mulungu-do-litoral [Erythrina speciosa (Fabales: Fabaceae)] (ZANuncio et al. 2015), grevílea [Grevillea robusta A. Cunn. Ex R. Br. (Proteales: Proteaceae)] (SANTANA et al. 2005), alfavaca-cravo [Ocimum gratissimum L. (Lamiales: Lamiaceae)] (RANDO \& LIMA 2010), açaizeiro [Euterpe oleracea Martius (Arecales: Arecaceae)] (SANTOS et al. 2015) e teca
[Tectona grandis Linn. f. (Lamiales: Verbenaceae)] (TAVAREs et al. 2018). Atualmente $A$. reticulatum está distribuída por quase todo o Brasil, podendo ser encontrada nos seguintes Estados: $\mathrm{AM}, \mathrm{AC}, \mathrm{AP}, \mathrm{BA}, \mathrm{CE}, \mathrm{ES}, \mathrm{DF}, \mathrm{GO}, \mathrm{MG}, \mathrm{PA}, \mathrm{PR}, \mathrm{RJ}, \mathrm{RS}, \mathrm{SC}$ e SP (MARques 1928; SANTANa et al. 2005; Jordão \& Silva 2006; BARONIO et al. 2012).

Formigas Camponotus (Hymenoptera: Formicidae) e abelhas Trigona (Hymenoptera: Apidae) foram encontradas em associação com as cigarrinhas neste estudo. A interação entre a formiga e a cigarrinha resultam numa relação de benefício mútuo entre as espécies, conforme já foi observado e relatado por FOWLER (1992) e ZANUNCIO et al. (2015) em Bauhinia forficata Link (Fabales: Fabaceae) e E. speciosa, respectivamente. Já a associação com Trigona não é vista como uma relação dependente, apesar de a abelha agir como um coletor da secreção resultante da alimentação das cigarrinhas e também defendê-la de possíveis predadores (VIEIRA et al. 2007; BARONIO et al. 2012). No presente trabalho, foi observada a ocorrência desses dois gêneros de himenópteros simultaneamente junto às cigarrinhas.

Diversos parasitoides já foram relatados em A. reticulatum, sobretudo insetos da ordem Hymenoptera, incluindo representantes de Myrmaridae, Trichogrammatidae e Encyrtidae (SiLVA et al. 1968), e também um tripes ectoparasita, Aulacothrips dictyotus Hood (Thysanoptera: Heterothripidae) (Izzo et al. 2002). Não foram encontrados inimigos naturais de A. reticulatum durante a execução deste estudo. Entretanto, este trabalho registra pela primeira vez a infestação de $A$. reticulatum em plantas de noni no Brasil.
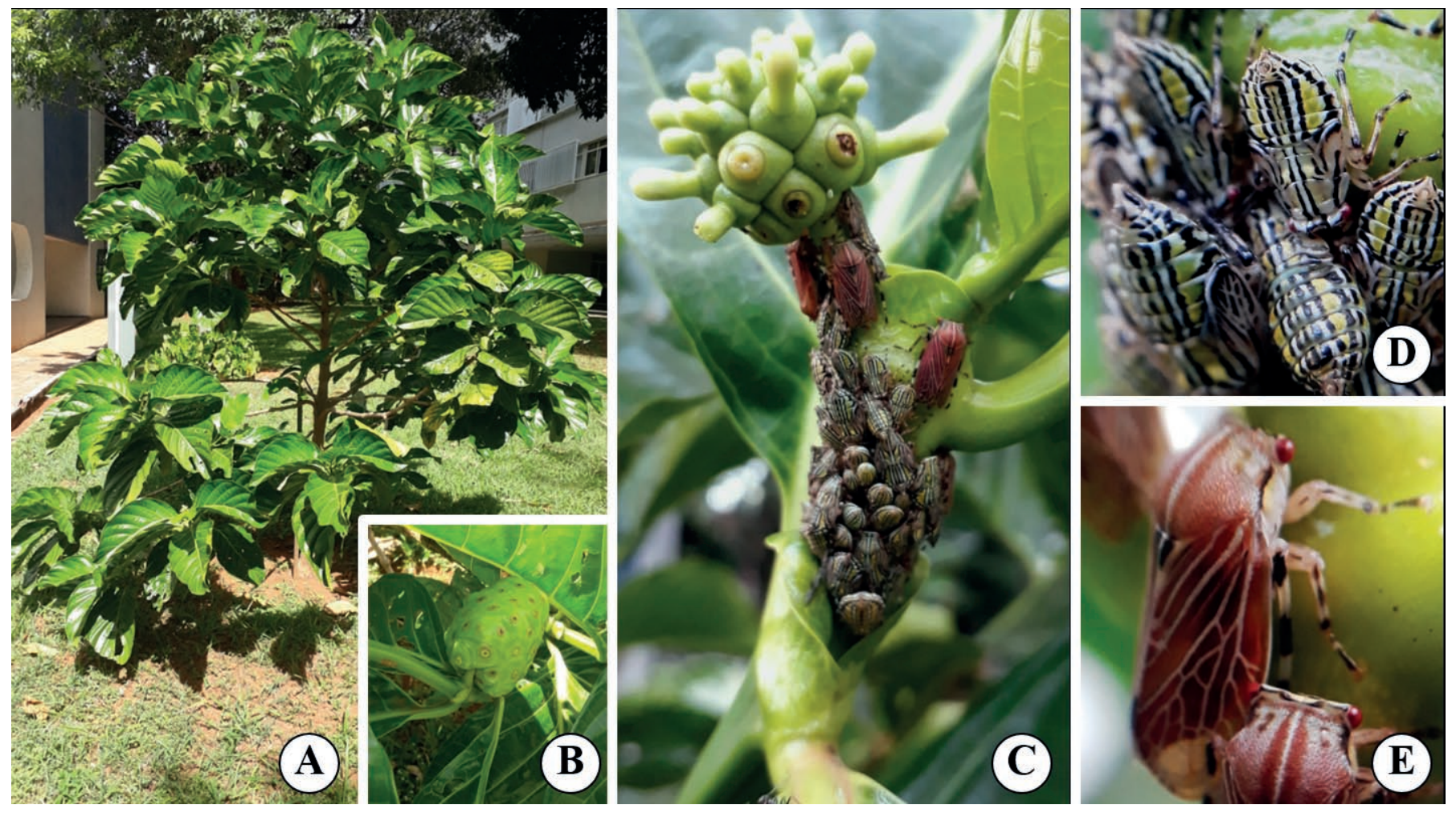

Figura 1. Aethalion reticulatum infestando plantas de noni (Morinda citrifolia) em Brasília, Brasil. A - Arbusto de noni em Brasília, Brasil. B Detalhe do fruto de noni. C - Colônia da cigarrinha em planta de noni. D - Ninfas de A. reticulatum. E - Adultos de A. reticulatum. (Foto: MT Castro).

\section{REFERÊNCIAS}

Baronio, GJ, ACV Pires \& C Aoki, 2012. Trigona branneri

(Hymenoptera: Apidae) as a collector of honeydew from Aethalion reticulatum (Hemiptera: Aethalionidae) on Bauhinia forficata (Fabaceae: Caesalpinoideae) in a Brazilian savanna. Sociobiology, 59: 407-414. DOI: https://doi.org/10.13102/sociobiology.v59i2.603.

Borror, DJ \& DM DeLong, 1969. Introdução ao estudo dos insetos. São Paulo: USP, 653 p.

Correia, AAS, MLC Gonzaga, AC Aquino, PHM Souza, RW

Figueiredo \& GA Maia, 2011. Caracterização química 
e físico-química da polpa do noni (Morinda citrifolia) cultivado no estado do Ceará. Alimentos e Nutrição, 22: 609-615.

Costa Lima, AM, 1942. Insetos do Brasil $-3^{\circ}$ Tomo: Homópteros. Escola Nacional de Agronomia (Série Didática n.4), 327 p.

Fowler, HG, 1992. Aetalionidae: functional equivalents of extrafloral nectaries in Bauhznza (Cesalpinionidea). Anales de Biología 18: 155-159.

Gallo, D, O Nakano, S Silveira-Neto, RPL Carvalho, GC Batista, E Berti Filho, JRP Parrra, RA Zucchi, SB Alves, JD Vendramim, LC Marchini, JRS Lopes \& C Omoto, 2002. Entomologia Agrícola. Piracicaba: FEALQ, 920 p.

Izzo, TJ, SMJ Pinent \& LA Mound, 2002. Aulacothrips dictyotus (Thysanoptera: Heterothripidae): the first ectoparasitic thrips (Thysanoptera). Florida Entomologist, 85: 281-283. DOI: https://doi.org/10.1653/0015-4040(2002)085[0281: adhtfe]2.0.co;2.

Jordão, AL \& RA Silva, 2006. Guia de pragas agrícolas para o manejo integrado no estado do Amapá. Ribeirão Preto: Holos, 183 p.

Marques, LAA, 1928. Cigarrinha nociva a várias espécies vegetais: biologia do membracídeo Aethalium reticulatum (L.). Instituto Biológico de Defesa Agrícola e Animal, Boletim 6, $27 \mathrm{p}$.

Matoso, LML, CCR. de Melo, LMDCS Menezes, LE de Oliveira \& KKD de Oliveira, 2013. As Características e a Utilização do Noni (Morinda citrifolia L.). Ciência \& DesenvolvimentoRevista Eletrônica da FAINOR, 6: 42-50.

Matte, WD, LF de Queiroz \& JN Corassa, 2016. Primeiro registro de Atta sexdens rubropilosa Forel, 1908 (Hymenoptera: Formicidae) atacando noni (Morinda citrifolia L.) em Colorado do Oeste (RO), Brasil. Arquivos do Instituto Biológico, 83: 1-4. DOI: https://doi.org/10.1590/18081657000722015 .

Nascimento, AS \& RS Carvalho, 1998. Pragas da mangueira. pp. 155-167. In: Sobrinho, NRB, JE Cardoso \& FCO Freire [eds.], Pragas de fruteiras tropicais de importância agroindustrial. Embrapa Agroindústria Tropical, Fortaleza, Brasil, 209 p.

Pimentel, D.D., A.M.B. Meira, C.R.F. de Araújo \& M.I. Peixoto, 2016. O uso de noni (Morinda citrifolia L.) por pacientes oncológicos: um estudo bibliográfico. Revista Saúde e Ciência online, 5: 37-44.

Rando, JSS \& CB Lima, 2010. Detecção de Aethalion reticulatum (L., 1717) (Hemiptera: Aethalionidae) em alfavaca-cravo (Ocimum gratissimum L.) e observações sobre sua ocorrência. Revista Brasileira de Plantas Medicinais, 12: 239-242. DOI: https://doi.org/10.1590/s151605722010000200018.

\section{Suggestion citation:}

de Castro, MT \& SCL Montalvão, 2019. Primeiro relato de Aetalion reticulatum (L.) (Hemiptera: Aetalionidae) infestando plantas de noni [Morinda citrifolia L. (Rubiaceae)]. EntomoBrasilis, 12 (2): 81-83. Available on: doi:10.12741/ebrasilis.v12i2.795
Santana, DLQ, CA Ferreira, EG Martins \& HD Silva, 2005. Ocorrência de Aethalion reticulatum (Linnaeus, 1767) (Hemiptera: Aethalionidae) em Grevillea robusta. Boletim de Pesquisa Florestal, 50: 109-115.

Santos, RS, AJ Creão-Duarte \& AMP Lunz, 2015. Infestação de Aetalion reticulatum (Linnaeus) (Hemiptera: Auchenorrhyncha: Aethalionidae) em Plantas de Euterpe oleracea Martius (Arecaceae) no Estado do Acre. EntomoBrasilis, 8: 69-73. DOI: http://dx.doi.org/10.12741/ ebrasilis.v8i1.450.

Silva, AGd'A, CR Gonçalves, DM Galvão, AJL Gonçalves, J Gomes, MN Silva \& L Simoni, 1968. Quarto catálogo dos insetos que vivem nas plantas do Brasil: seus parasitos e predadores. Rio de Janeiro, Ministério da Agricultura. 622 p.

Silva, LR, PVQ Medeiros, GA Leite, KJP Silva, CV Mendonça \& GG Silva, 2012. Caracterização do fruto de Morinda citrifolia L. (noni). Revista Cubana de Plantas Medicinales, Ciudad de la Habana, 17: 93-100.

Solomon, N, 1999. O fruto tropical de 101 aplicações medicinais: Suco de Noni. 1 a ed. Vineyard: Utah. 31 p.

Sousa, JA, ARL Aquino, FCO Freire \& PAF Silva Neto, 2010. Produção de mudas de noni (Morinda citrifolia L.). Comunicado Técnico 157, Embrapa Agroindústria Tropical, $4 \mathrm{p}$.

Tavares, TA, AG Fonseca, FF Sousa \& SL de Assis Júnior, 2018. Ocorrência de Aethalion reticulatum Linnaeus (Hemiptera: Aethalionidae) em Tectona grandis Linn. f. (Verbenaceae) em Minas Gerais, Brasil. EntomoBrasilis, 11: 220-222. DOI: https://doi.org/10.12741/ebrasilis.v11i3.752.

Tombolato, AFC, W Barbosa \& R Hiroce, 2005. Noni: Frutífera medicinal em introdução e aclimatação no Brasil. Informações técnicas: O agronômico, 57: 20-21.

Vieira, CU, CM Rodovalho, LO Almeida, ACS Siquieroli \& AM Bonetti, 2007. Interação entre Trigona spinipes Fabricius, 1793 (Hymenoptera: Apidae) e Aethalion reticulatum Linnaeus, 1767 (Hemiptera: Aethalionidae) em Mangifera indica (Anacardiaceae). Bioscience Journal, Supplement 1, 23: 10-13.

Wang, MY, BJ West, CJ Jensen, D Nowicki, C Su, AK Palu, \& G Anderson, 2002. Morinda citrifolia (Noni): A literature review and recent advances in Noni research. Acta Pharmacologica Sinica, 12: 1127-1141.

Zanuncio, AJV, JE Serrão, AIA Pereira, MA Soares, CF Wilcken, GLD Leite \& JC Zanuncio, 2015. Aethalion reticulatum (Hemiptera: Aethalionidae) Feeding on Erythrina speciosa (Fabales: Fabaceae): First Record of Its Host Plant and Damage Characteristics. Florida Entomologist, 98: 175-177. DOI: https://doi.org/10.1653/024.098.0130.

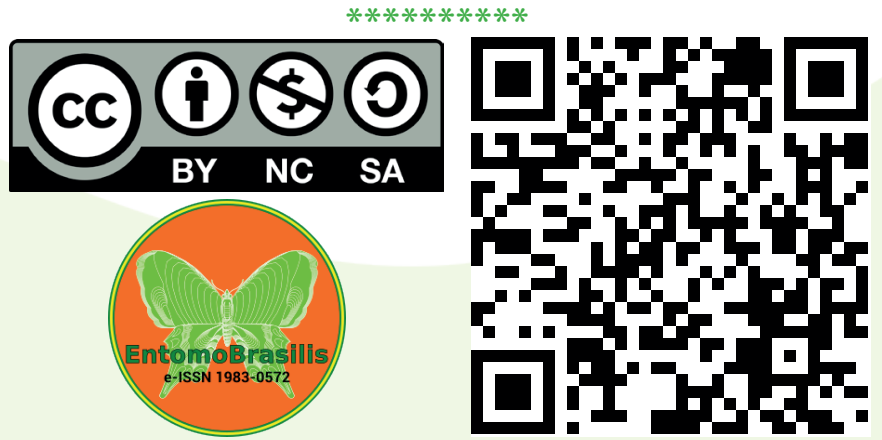

